\title{
FINITE TYPE CONES SHAPED ON SPHERICAL SUBMANIFOLDS
}

\author{
OSCAR J. GARAY
}

(Communicated by David Ebin)

\begin{abstract}
The only finite type cones shaped on spherical submanifolds are minimal ones.
\end{abstract}

1. Introduction. Submanifolds of finite type introduced in [1] can be considered as a generalization of minimal submanifolds of a sphere and minimal submanifolds of an Euclidean space. A submanifold $M$ of an Euclidean space $E^{m+1}$ is said to be of $k$-type if the position vector $x$ of $M$ in $E^{m+1}$ can be expressed in the following form:

$$
x=c+x_{i_{1}}+\cdots+x_{i_{k}}, \quad \Delta x_{i_{t}}=\lambda_{i_{t}} x_{i_{t}}, \lambda_{i_{1}}<\cdots<\lambda_{i_{t}},
$$

where $c$ is a constant vector; $\lambda_{i_{t}} \in \mathbf{R}$ are constant and, $\Delta$ is the Laplacian of the submanifold $M$ with respect to the induced metric. For a $k$-type submanifold $M$, there exists a polynomial $P(t)$ of degree $k$ such that $P(\Delta) H=0$, where $H$ denotes the mean curvature vector of $M$ in $E^{m+1}$. (See $[1,2]$ for more details.)

For a compact submanifold $M$ of the unit hypersphere $S^{m}$ of radius 1 centered at the origin, J. Simons [3] proved that if $M$ is minimal in $S^{m}$, then $C M-\{0\}$ is minimal in $E^{m+1}$, where $C M$ denotes the cone over $M$. In terms of finite type terminology, Simons' result says that $C M-\{0\}$ is of 1-type when $M$ is of 1-type.

In this article, we prove the following

THEOREM. Let $x: M \rightarrow S^{m}$ be an isometric immersion of a compact Riemannian manifold $M$ into $S^{m}$. Then the punctured cone $C M-\{0\}$ is of finite type if and only if $M$ is minimal in $S^{m}$.

2. Lemmas. Let $M$ be a compact $p$-dimensional submanifold of $S^{m}$. The cone over $M, C M$, is defined by the following map: $M \times[0,1] \rightarrow E^{m+1} ;(m, t) \rightarrow t m$. Let $H$ and $H^{\prime}$ denote the mean curvature vectors of $C M-\{0\}$ in $E^{m+1}$ and that of $M$ in $S^{m}$, respectively, and denote by $\nabla$ and $\nabla^{\prime}$ the connections of $E^{m+1}$ and $M$. For $m \in M$ we choose a local orthonormal frame $\left\{E_{i}\right\}_{i=1}^{p}$ such that $\nabla_{E_{i}}^{\prime} E_{j}(p)=0$. Let $\xi$ be the unit vector field of $C M$ given by $\partial / \partial t$. Then the integral curves of $\xi$ are rays from the origin of $E^{m+1}$. By extending the fields $\left\{E_{i}\right\}_{i=1}^{p}$ along the rays via parallel translation, we obtain an orthonormal local frame field on $C M$, which we also denote by $\left\{E_{i}\right\}_{i=1}^{p}$.

If $\sigma$ is the second fundamental form of $M$ in $S^{m}$, then we have

$$
\nabla_{E_{i}} E_{i}(m, t)=\left(1 / t^{2}\right) \sigma\left(E_{i}, E_{i}\right)(m)-(1 / t) \xi .
$$

Received by the editors September 10, 1987.

1980 Mathematics Subject Classification (1985 Revision). Primary 53C40. 
So we have

LEMMA 1. Let $x: M \rightarrow S^{m}$ be an isometric immersion of a closed Riemannian manifold $M$ into $S^{m}$, and $C M-\{0\}$ the punctured cone over $M$. Then we have

$$
H(m, t)=\left(p /(p+1) t^{2}\right) H^{\prime}(m) .
$$

ProOF. Because $\nabla_{\xi} \xi=0,(2.1)$ implies

$$
H(m, t)=\left(\frac{1}{(p+1)}\right)\left\{\sum_{i=1}^{p} \nabla_{E_{i}} E_{i}+\nabla_{\xi} \xi\right\}^{N}=\left(\frac{1}{(p+1) t^{2}}\right)\left\{p H^{\prime}-p t \xi\right\}^{N},
$$

where $(,)^{N}$ denote the normal component. So one has (2.2). Q.E.D.

Now, we compute the Laplacian of the cone in terms of the Laplacian of $M$. We shall see that Lemma 6.1.2 of [3] remains valid without the minimality condition on $M$.

LEMMA 2. Under the hypothesis of Lemma 1, for a smooth function $f$ of $C M-$ $\{0\}$ we have

$$
(\bar{\Delta} f)(m, t)=\left(\frac{1}{t^{2}}\right) \Delta f_{t}(m)-\left\{\frac{\partial^{2} f}{\partial t^{2}}(m, t)+\frac{p}{t} \frac{\partial f}{\partial t}(m, t)\right\},
$$

where $\bar{\Delta}$ and $\Delta$ denote the Laplacians of $C M-\{0\}$ and $M$, respectively, and $f_{t}$ is defined by $f_{t}(m, t)=f(m, t), t \in(0,1]$.

Proof. We choose $\left\{E_{i}\right\}_{i=1}^{p}$ and $\xi$ as before. Then we get

$$
E_{i}(f)(m, t)=(1 / t) E_{i}\left(f_{t}\right)(m) .
$$

On the other hand, if $\nabla_{\xi}^{\prime \prime} \xi$ is the Riemannian connection of $C M-\{0\}$, then

$$
\bar{\Delta} f(m, t)=-\left\{\xi \xi(f)-\left(\nabla_{\xi}^{\prime \prime} \xi\right)(f)+\sum_{i=1}^{p}\left[E_{i} E_{i}(f)-\nabla_{E_{i}}^{\prime \prime} E_{i}(f)\right]\right\}
$$

and so by using (2.1) and (2.4) we have (2.3). Q.E.D.

Now, we put

$$
a_{n}=2 n(p-2 n-1), \quad n=1,2, \ldots,
$$

where $p$ is the dimension of $M$. Let $S_{J}\left(x_{1}, \ldots, x_{n}\right)$ denote the elementary symmetric functions in $x_{1}, \ldots, x_{n}$, i.e.,

$$
S_{1}\left(x_{1}, \ldots, x_{n}\right)=\sum_{i=1}^{n} x_{i}, S_{2}\left(x_{1}, \ldots, x_{n}\right)=\sum_{i<j} x_{i} x_{j}, \ldots, S_{n}\left(x_{1}, \ldots, x_{n}\right)=x_{1} \cdots x_{n} .
$$

We also put $d_{J}^{n}=S_{J}\left(a_{1}, \ldots, a_{n}\right)$. Then we have

LEMMA 3. If $x: M \rightarrow S^{m}$ is an isometric immersion of a compact Riemannian manifold $M$ into $S^{m}$, then for any integer $n$ we have

$\bar{\Delta}^{n} H=\left(p /(p+1) t^{2(n+1)}\right)\left\{\Delta^{n} H^{\prime}+d_{1}^{n} \Delta^{n-1} H^{\prime}+d_{2}^{n} \Delta^{n-2} H^{\prime}+\cdots+d_{n-1}^{n} \Delta H^{\prime}+d_{n}^{n} H^{\prime}\right\}$. 
ProOF. We put $H=\left(h_{1}, \ldots, h_{m+1}\right)$ and $H^{\prime}=\left(h_{1}^{\prime}, \ldots, h_{m+1}^{\prime}\right)$. Then from (2.2) we have

$$
h_{i}=\left(p /(p+1) t^{2}\right) h_{i}^{\prime}, \quad i=1, \ldots, m+1 .
$$

By using (2.3) one gets

$$
\bar{\Delta}\left(h_{i}\right)=\left(p /(p+1) t^{4}\right)\left\{\Delta h_{i}^{\prime}+(2 p-6) h_{i}^{\prime}\right\}, \quad i=1, \ldots, m+1,
$$

so that

$$
\bar{\Delta} H=\left(p /(p+1) t^{4}\right)\left\{\Delta H^{\prime}+(2 p-6) H^{\prime}\right\} .
$$

We repeat the argument on the component functions of $\bar{\Delta} H$ to obtain

$$
\bar{\Delta}^{2} H=\left(p /(p+1) t^{6}\right)\left\{\Delta^{2} H^{\prime}+[4(p-5)+2(p-3)] \Delta H^{\prime}+8(p-5)(p-3) H^{\prime}\right\} .
$$

Hence, (2.6) follows by induction. Q.E.D.

3. Proof of Theorem. Suppose there exists a minimal polynomial of degree $k, P(t)=t^{k}+c_{1} t^{k-1}+\cdots+c_{k}$, such that

$$
\bar{\Delta}^{k} H+c_{1} \bar{\Delta}^{k-1} H+\cdots+c_{k} H=0, \quad c_{i} \in \mathbf{R} .
$$

Then, by substituting (2.6) into (3.1), we have

$$
\begin{gathered}
\Delta^{k} H^{\prime}+\left(d_{1}^{k}+c_{1} t^{2}\right) \Delta^{k-1} H^{\prime}+\left(d_{2}^{k}+c_{1} d_{1}^{k-1} t^{2}+c_{2} t^{4}\right) \Delta^{k-2} H^{\prime} \\
+\cdots+\left(d_{k}^{k}+c_{1} d_{k-1}^{k-1} t^{2}+\cdots+c_{k-1} d_{1}^{1} t^{2 k-2}+c_{k} t^{2 k}\right) H^{\prime}=0
\end{gathered}
$$

Since equality (3.2) holds for every $(m, t)$ in $C M-\{0\},(3.2)$ is true for any $t$ in $(0,1]$ at every fixed point $m$ in $M$. Therefore, we conclude that $H^{\prime}=0$. The converse follows from (2.2). Q.E.D.

\section{REFERENCES}

1. B. Y. Chen, Total mean curvature and submanifolds of finite type, World Scientific, 1984.

2. _ Finite type submanifolds and generalizations, University of Rome, 1985.

3. J. Simons, Minimal varieties in riemannian manifolds, Ann. of Math. 88 (1968), 62-105.

Departamento de Geometria y Topologia, Facultad de Ciencias, UniversiDAD DE GRANADA, 18002-GRANADA, SPAIN 